\title{
A Hybrid Approach to Prototyping and Testing: Combining Physical and Virtual Reality to Connect Users and Designers
}

\author{
Yi Lin Wonga*, Chi Hang Lo ${ }^{b}$ \\ aThe Hong Kong Polytechnic University

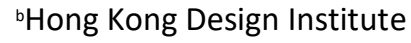 \\ *Corresponding author e-mail: elaine-yl.wong@polyu.edu.hk
}

\begin{abstract}
:
During the COVID-19 pandemic, various social distancing measures have been implemented worldwide. These measures have delayed design projects by prohibiting face-to-face interaction between designers and users and preventing users from physically touching prototypes. Virtual reality (VR) technology may offer a solution to this problem. To support design projects during the COVID-19 pandemic, this paper presents an approach to user evaluation that combines physical and virtual prototyping. Users can test design solutions and offer feedback both in person by visiting design studios and remotely using VR technology. The paper discusses the benefits and limitations of this hybrid approach for stakeholders in design projects.
\end{abstract}

Keywords: virtual reality; prototyping; testing; social distancing measures

\section{Introduction}

During the COVID-19 pandemic, many design projects have been delayed. The progress of co-design projects has been particularly slow, as such projects involve considerable interaction between designers and users. Designers need to obtain continuous feedback from a range of users to optimise their designs. However, such extensive contact increases the risk of infection, and designers may even carry the virus from one user group to another. In response to the social distancing measures enacted during the pandemic, design teams have begun to seek strategies for contactless interaction with users. Video conferencing software such as Zoom and Microsoft Teams is used in many industries to obtain verbal feedback through discussion and interviews. However, in the design context, users have to experience a prototype before they can provide feedback on it. Social distancing measures not only limit face-to-face interaction between designers and users but also prevent users from physically touching and using design prototypes.

Virtual reality (VR) technology can offer an alternative to face-to-face interaction and physical contact with prototypes during a design project. If a real product is not available, users can test a 
virtual substitute. However, VR testing may not be equivalent to physical testing, as designers can choose what is included in the virtual environment (Whyte \& Nikolić, 2018). For instance, when users are asked to evaluate a piece of furniture using VR, the virtual set-up may not include key attributes of the environment, such as the size of the room in which the furniture is located. Therefore, care must be taken when using VR for product evaluation. Nevertheless, many designers and researchers have advocated the use of VR to involve users in the design process, as this approach can reduce costs and provide a platform for users and designers to smoothly exchange information on the design process (Bruno \& Muzzupappa, 2010; Rebelo, Duarte, Noriega, \& Soares, 2011; Svidt \& Sørensen, 2016). Through its immersive and interactive functions, VR reduces the chance of misunderstanding between users and designers and helps users to understand the immediate consequences of their design decisions (Thalen \& van der Voort, 2012).

Numerous studies have explored the use of VR to involve users in the design process. Serrano, Botella, Baños, and Alcañiz (2013) examined a virtual environment in which users could view and test ceramic tile products. The interface allowed the users to see how the tiles might look in various realworld settings. The users reported high levels of satisfaction and relaxation and a strong sense of presence during the virtual testing process. Grajewski, Górski, Zawadzki, and Hamrol (2013) applied VR technology to the design of manufacturing workplaces. They built virtual prototypes that enabled users to interact remotely with the workplaces. In addition to testing the objects in the workplaces and their spatial arrangements, the researchers performed collision detection and kinematics testing using VR technology. Bruno and Muzzupappa (2010) used VR to create a virtual product interface in a participatory design context. To assess its effectiveness, they compared the results of usability tests involving real and virtual products. They also tested the feasibility and effectiveness of assigning an operator to help users interact with the virtual interface and involving users as co-designers in the virtual environment. They found that VR was a valid and effective method of improving usability and involving users in the design process in various ways. Falcaõ and Soares (2013) also commented that VR enhanced usability. In sum, many design researchers have reported that VR offers a promising approach to prototyping and testing. However, most studies in this area have focused on VR alone; very few have considered how physical and virtual prototyping can be adopted concurrently in a design project.

To support design teams in today's era of social distancing, this paper presents a hybrid approach to user evaluation that involves both physical and virtual prototyping. This approach allows users to engage with and give feedback on design solutions not only in person but also virtually via VR technology. The paper discusses the benefits and limitations of this approach for stakeholders in design projects.

\section{Design Project: Original Plan}

To illustrate how VR technology can be applied to obtain feedback on the design process, this paper describes a design project focusing on living space, lighting, and furniture in subdivided units (SDUs) in Hong Kong. According to the Hong Kong Census and Statistics Department (C\&SD, 2016), SDUs are created when 'domestic quarters are subdivided into two or more smaller units for rental purposes' (p. 11). SDU residents thus sacrifice living space in exchange for lower rent. Some units do not even have their own bathrooms or kitchens, as tenants must share these facilities with other households. Most SDUs are located in urban areas of Hong Kong with high poverty rates (Leung \& Yiu, 2019).

The original aim of the project was to redesign furniture and modify the use of space and lighting in SDUs to improve tenants' quality of life. Children were selected as the main user group in the pilot 
project, as children living in SDUs are most severely affected by the limitations of their living environment. First, children living in these units do not have a quiet environment in which to do their homework, as all family members use the same main space; there is no differentiation between living, dining, and study areas. This is a major barrier to children's learning. Second, as there is not enough space for different kinds of furniture in SDUs, children have to use foldable tables as desks and sit on stools that are too high for them. Because of their low socioeconomic status, most families living in SDUs are unable to afford furniture that is ergonomically designed for children's body shapes and sizes.

The designers and researchers involved in the project conducted field visits to SDUs and obtained data on the living environments of three representative families. Photographs were taken of the interior spaces of the families' SDUs. Figure 1 below shows one such photograph.

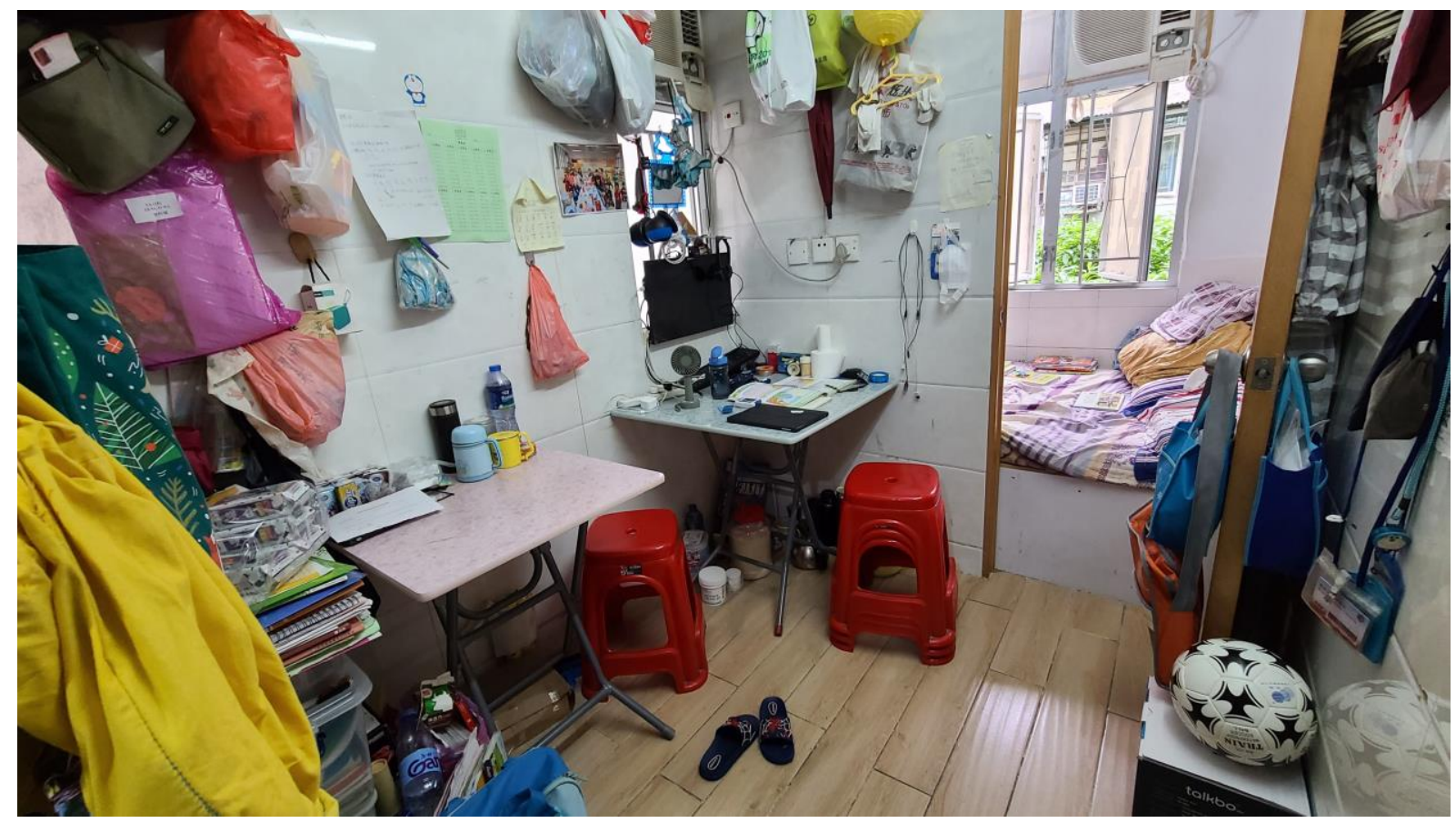

Figure 1. Interior of a subdivided unit in which one of the participating families lived (photograph taken by the authors). All family activities except sleeping took place in the main area shown here. The foldable table in the corner of the room was used by the children as a desk for studying. The other foldable table was used as the family's dining table. Miscellaneous items had been hung on the walls in plastic or non-woven bags.

Next, design thinking workshops were conducted with design experts and social workers to identify and suggest solutions to shortcomings in the families' living environments. The insights gained in these workshops helped the designers to consolidate their ideas for redesigning the units' furniture lighting and living spaces. Originally, they intended to build full-size models of the redesigned SDUs and invite the participating families to visit the models and comment on their spatial arrangements, lighting, and furniture. However, because of restrictions imposed by the COVID-19 pandemic, the families would have been unable to interact with the SDU models in person. An alternative plan was needed.

\section{Alternative Plan: Using Virtual Reality}

After thorough discussion, the members of the design team decided to use VR technology to enable the families to experience the redesigned SDUs while complying with social distancing measures. 
Instead of creating full-size physical models, the team used the computer software tool Enscape to create three-dimensional graphical models of the SDUs, producing VR computer files (as shown in Figure 2) that could be opened in the smartphone app Google Cardboard. A VR smartphone headset was sent to each of the families by post. The families were asked to download the smartphone app and VR files and use the headsets to view the redesigned SDUs in a VR environment. The costeffective VR smartphone headsets were either devices such as that shown in Figure 3 or easily assembled cardboard devices (EETech, n.d.). Figure 4 shows how the headsets were worn.

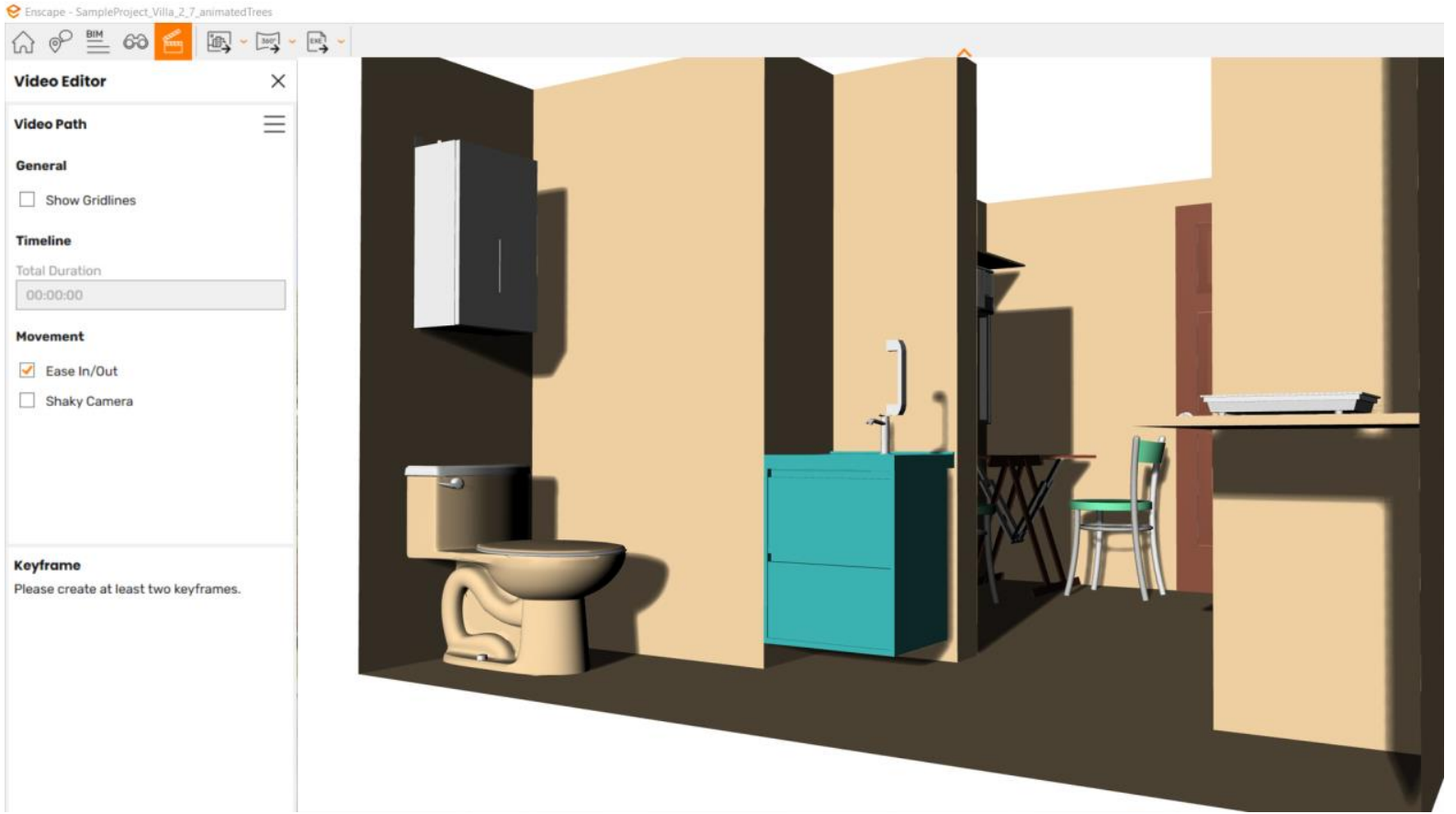

Figure 2. Interface of the computer software Enscape, showing one of the modified SDU designs. These designs were exported to the VR smartphone app Cardboard.

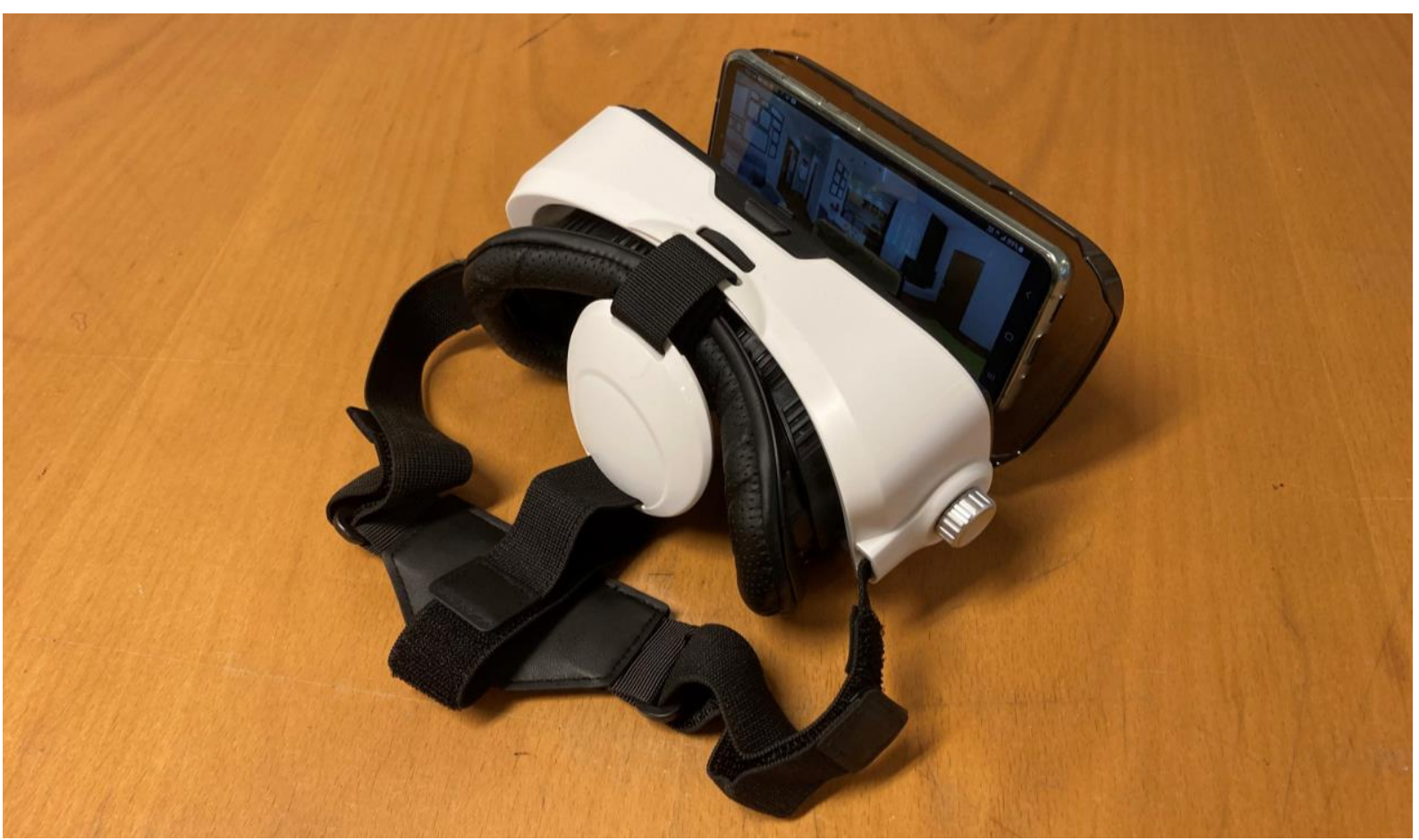

Figure 3. One of the VR headsets sent to the participating families. A smartphone was put inside each headset. Users entered the virtual environment using the Cardboard app and Enscape files (Photograph taken by the authors). 


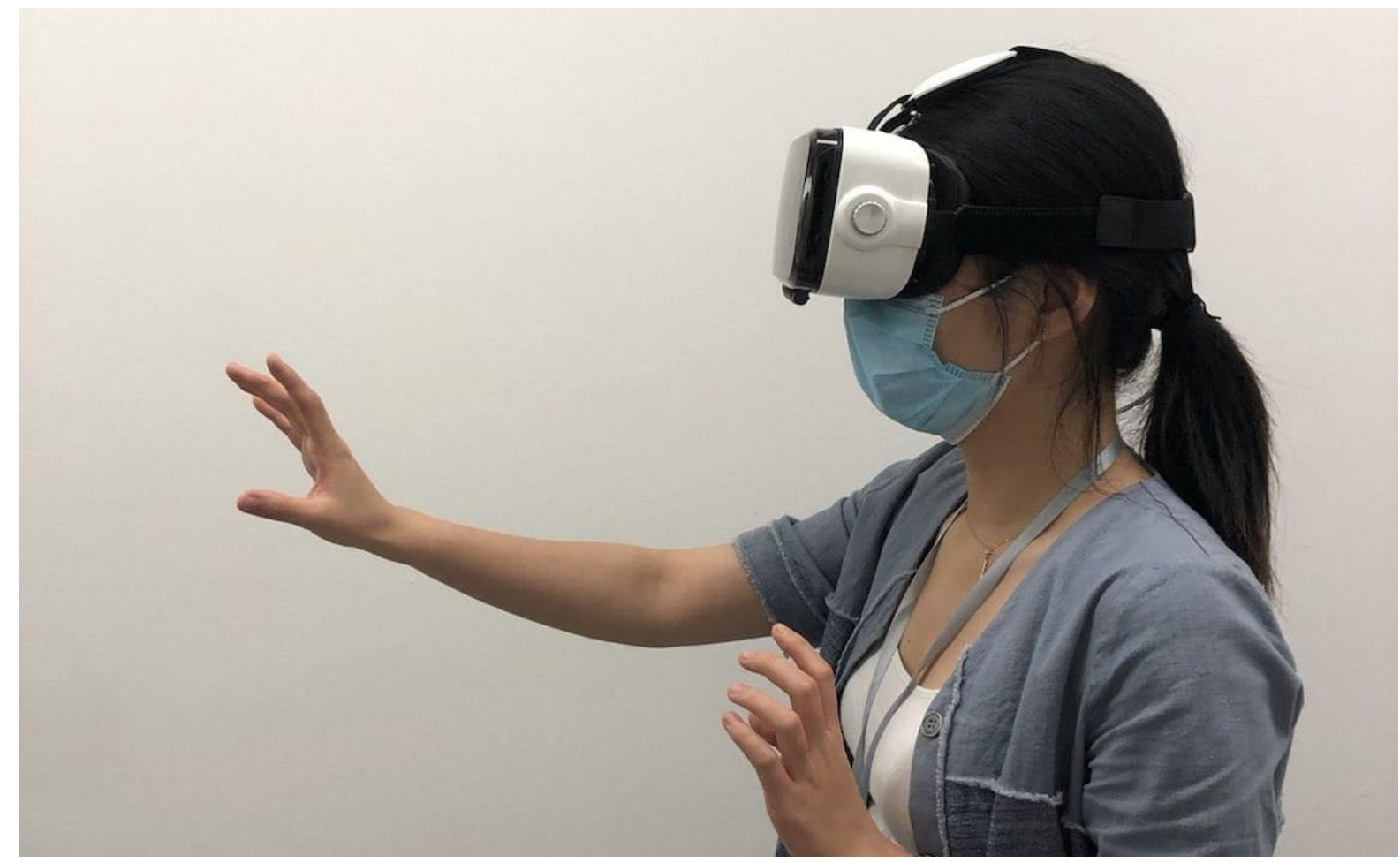

Figure 4. Demonstration of the use of a VR smartphone headset (photograph taken by the authors).

The children in the participating families explored the modified SDUs virtually, with three or four designs for each unit. An online questionnaire survey was designed to collect their feedback on the lighting, spatial arrangements, furniture, and other aspects of the redesigned units. As the VR tests were performed in the participants' homes and the VR smartphone headsets had certain limitations, the children were unable to walk around to see every detail of each design. Their field of vision was 360 degrees but was limited to a single position. In addition, they could not interact with the VR environment.

Next, based on the data collected from the completed questionnaires, the designs were refined using Enscape. In addition, full-size physical models of the redesigned furniture were created. The families were then invited to the designers' studio to test the new designs. The children were each given an Oculus all-in-one VR headset and two touch controllers to enable them to fully immerse themselves in the VR environment. In addition to viewing full-size physical models of the redesigned furniture, the participants could walk around the VR environment to holistically experience the final SDU designs. VR technology supports the evaluation of ergonomics, as suggested by Aromaa and Väänänen (2016). The participants evaluated the ergonomic qualities of the redesigned furniture, spatial arrangements, and lighting. They were asked to try placing books, stationery, and other objects on their desks in the VR environment to explore how the redesigned furniture could be used. This interaction was made possible by the use of Oculus all-in-one VR headsets.

During this process, the children and other family members were also asked to provide feedback on the designs. Based on their feedback, the designers further modified the designs in real time by changing the design parameters using computer software, and the participants provided more feedback after each modification. This process was repeated until the participants were satisfied with the final designs. 


\section{Discussion}

The COVID-19 pandemic has profoundly changed our lives. It has also changed the ways in which research can and should be conducted. Design scholars now have the opportunity to rethink and transform the process of design research. The pandemic has advanced the application of VR technology in many areas, and design research is undoubtedly one of them. The progress of the SDU design project described here was impeded by COVID-19 social distancing measures, requiring alternative arrangements to be made. Unexpectedly, the new arrangements optimised the design process and yielded important insights into virtual prototyping and testing. Figure 5 below shows the original plan for the project and the alternative plan developed to comply with social distancing measures during the pandemic. The paragraphs below discuss the advantages of the alternative plan.

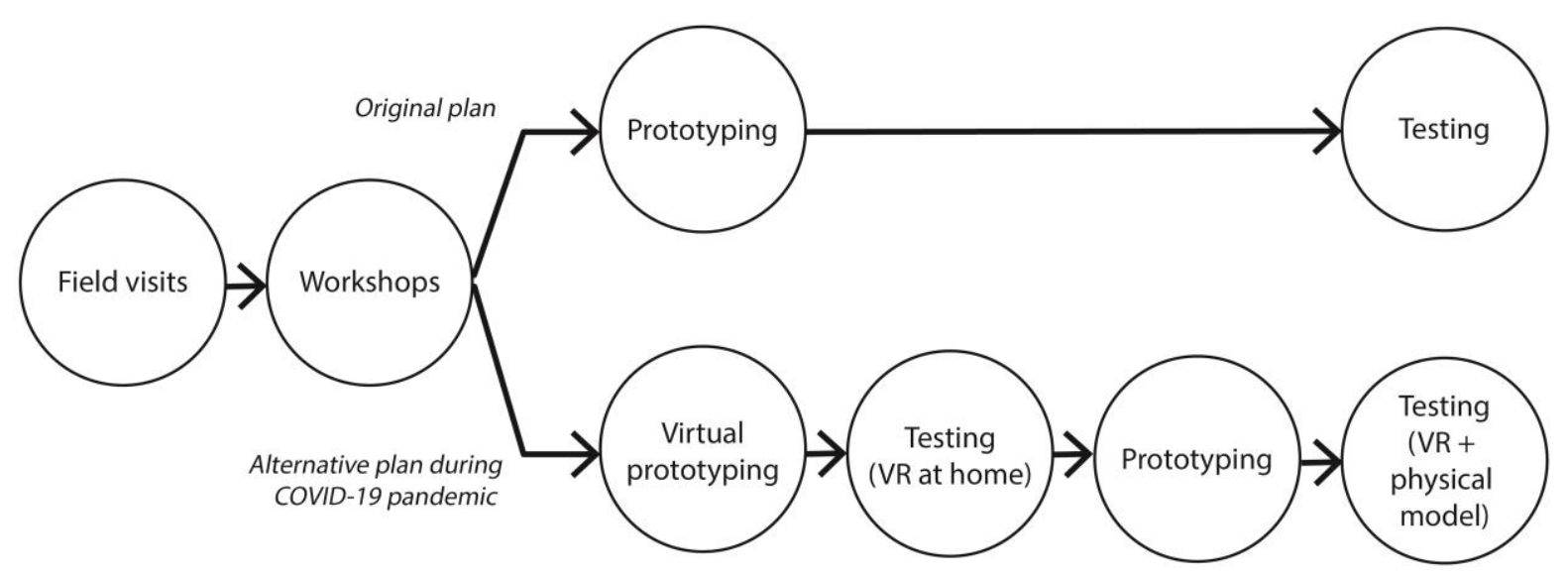

Figure 5. Original plan for project and alternative plan to comply with social distancing measures during the COVID-19 pandemic.

The alternative plan added a cycle of VR prototyping and testing to the project. This was performed by the participants in their own homes, independently of the designers. This significantly increased the users' active involvement and participation in the design process, which in turn enhanced their sense of ownership of the designs and helped them imagine how the redesigned units would look and function in real life (Scariot, Heemann, \& Padovani, 2012). Although the VR technology used in this cycle was not interactive, it was sufficient to enable the users to preview the designs developed for them. The opportunity to use this cutting-edge technology also increased the users' interest in testing the designs and completing the questionnaires and motivated them to take the process more seriously.

Implementing the alternative plan also reduced costs. Originally, the team intended to build full-size SDU models for the families to evaluate in person. This process would have been straightforward. However, much time, money, and effort would have been needed to modify the models based on the users' feedback for the second round of testing. In the final project, no physical SDU models were made. Davies (2004) similarly adopted VR for participatory design because VR design parameters can be easily changed and modifications can be easily recorded (Ferrise, Bordegoni, \& Cugini, 2013). In the study reported here, the use of virtual models not only dramatically reduced costs but also minimised the physical space needed for the project. As designing and making virtual SDU models proved much faster than designing and making physical SDU models, the development and manufacturing time was significantly shortened (Ottosson, 2002). The only challenge was recruiting designers who were familiar with VR technology to join the design team. 
The first cycle of prototyping and testing influenced the second cycle. Both virtual and physical models were used in the second stage of testing. In the VR environment at home, the users were unable to assess tactile attributes such as the weight of the furniture or the textures of the materials used; they could only experience the designs visually. To overcome this limitation and allow the users to experience the designs more holistically, the design team subsequently implemented simultaneous physical and VR testing.

In the final stage of testing, the designers further modified the designs instantly according to feedback provided by the children and other family members. This enriched the interaction between the users and designers. Therefore, although the original VR environment did not allow the users to interact with the models, this limitation ultimately enhanced the communication and exchange between the users and designers. The use of both VR and physical models provided an excellent platform for the users and designers to discuss design possibilities.

In most studies of the use of VR in design (e.g., Bruno \& Muzzupappa, 2010; Grajewski, Górski, Zawadzki, \& Hamrol, 2013; Serrano, Botella, Baños, \& Alcañiz, 2013), testing, evaluation, and feedback sessions have involved both designers/researchers and users and used a fully immersive virtual environment. Designers have normally led these sessions. In contrast, the users involved in this project used a less immersive VR device, i.e., a VR smartphone headset, in their own homes, without being observed by the designers. This may have made the users feel more relaxed, reducing the tension that can arise when participants are observed by figures of authority, such as designers. Such tension may have been particularly strong for the users involved in this study, as families living in SDUs usually have low socio-economic status. The user-independent, designer-absent experience helped the users to express their ideas freely. In this way, VR strengthened the user-centred nature of the design process. This finding contributes to research on user involvement in the design process (Rebelo et al., 2011).

Although the hybrid approach described here addressed key design challenges arising during the COVID-19 pandemic, it has some limitations that may have reduced the smoothness and quality of the prototype testing process. First, not all of the users may have been technologically literate. 'Technological literacy' refers to 'the ability to use, manage, assess, and understand technology' (International Technology Education Association, 2007, p. 9). Some of the participants may have been unable to use the smartphone app or download the Enscape files to their smartphones. In future, clear instructions for the participating families will be needed to ensure the effectiveness of their use of VR technology at home. Second, the participants were unable to interact with the VR environment at home. Such interaction would have required more advanced and thus much more expensive VR headsets and controllers, which could not feasibly have been sent to the participating families. This limited the users' experience during the first prototyping and testing cycle. However, the second prototyping and testing cycle did give the users the opportunity to interact with the VR environment, maximising the benefits of using VR technology. Combining visual and haptic modalities helps users to accurately evaluate aesthetic quality, functional features, and usability (Bordegoni \& Ferrise, 2012).

\section{Conclusions}

This paper shows how a design team adapted to social distancing measures during the COVID-19 pandemic by developing a hybrid approach to user evaluation that involved both VR technology and physical prototypes. As the project addressed the use of space, lighting, and furniture in SDUs, it was crucial to use physical as well as virtual methods, as the users needed to assess not only visual but 
also other sensory attributes, such as texture and weight. Adopting VR technology added a cycle of virtual prototyping and testing to the project. This was found to increase the users' sense of ownership of and participation in the design process. Communication between the users and designers was enhanced through the instant modification of the designs based on feedback in the final testing stage. As some of the prototypes were presented in virtual space, fewer materials were used in the prototyping process, which reduced the cost and duration of development and manufacturing. However, the hybrid approach presented here has certain limitations that may have reduced the efficiency and effectiveness of the testing process. First, more support may be needed for families who are not technologically literate. Second, the users' VR experience at home lacked interactivity.

Further research should collect more feedback from users on their VR experiences of design. Researchers could compare user evaluation using physical models only, using virtual models only, and using both physical and virtual models. Factors such as age, educational background, and technological literacy could be introduced to investigate their potential effects on user evaluation using VR technology. Measures of emotion could also be included to further assess users' engagement and satisfaction with the VR testing process.

\section{References}

Aromaa, S., \& Väänänen, K. (2016). Suitability of virtual prototypes to support human factors/ergonomics evaluation during design. Applied Ergonomics, 56, 11-18.

Bordegoni, M., \& Ferrise, F. (2012). Designing interaction with consumer products in a multisensory virtual reality environment. Virtual and Physical Prototyping, 8(1), 51-64.

Bruno, F., \& Muzzupappa, M. Product interface design: A participatory approach based on virtual reality. International Journal of Human-Computer Studies, 68(5), 254-269.

Census and Statistics Department (C\&SD), Hong Kong Special Administrative Region. (2016a). Thematic household survey report no. 60. Retrieved 3 May 2021 from http://www.statistics.gov.hk/pub/B11302602016XXXXB0100.pdf

Davies, R. C. (2004). Adapting virtual reality for the participatory design of work environments. Computer Supported Cooperative Work, 13, 1-33.

EETech. (n.d.). Build your own DIY Google cardboard-based virtual reality headset at home. Retrieved 3 May 2021 from https://maker.pro/custom/projects/diy-virtual-reality-headset

Falcaõ, C. S., \& Soares, M. M. (2013). Application of virtual reality technologies in consumer product usability. In A. Marcus (Ed.), Proceeding of DUXU/HCII 2013 Part IV (pp.342-351). Berlin, German: Springer-Verlag.

Ferrise, F., Bordegoni, M., \& Cugini, U. (2013). Interactive virtual prototypes for testing the interaction with new products. Computer-Aided Design and Application, 10(3), 515-525.

Grajewski, D., Górski, F., Zawadzki, P., \& Hamrol, A. (2013). Application of virtual reality techniques in design of ergonomic manufacturing workplaces. Procedia Computer Science, 25, 289-301.

International Technology Education Association. (2007). Standards for technological literacy. Retrieved 3 May 2021 from https://www.iteea.org/File.aspx?id=67767\&v=b26b7852

Leung, K. M., \& Yiu, C. Y. (2019). Rent determinants of sub-divided units in Hong Kong. Journal of Housing and the Built Environment, 34, 133-151.

Ottosson, S. (2002). Virtual reality in the product development process. Journal of Engineering Design, 13(2), 159-172.

Rebelo, F., Duarte, E., Noriega, P., \& Soares, M. M. (2011). Virtual reality in consumer product design: Method and applications. In W. Karwowski, M. M. Soares, \& N. A. Stanton (Eds.), Human factors 
and ergnomics in consumer product design: Methods and techniques (pp. 381-402). Baca Raton, FL: CRC Press, Taylor \& Francis Group.

Scariot, C. A., Heemann, A., \& Padovani, S. (2012). Understanding the collaborative-participatory design. Work, 41(sup.1), 2701-2705.

Serrano, B., Botella, C., Baños, R. M., \& Alcañiz, M. (2013). Using virtual reality and mood-induction procedures to test products with consumers of ceramic tiles. Computers in Human Behavior, 29(3), 648-653.

Svidt, K., \& Sørensen, J. B. (2016). Development of a virtual reality solution for end user involvement in interior design. In A. Herneoja, T. Österlund, \& P. Markkanen (Eds.), Complexity and simplicityProceedings of the $34^{\text {th }}$ eCAADe (Education and Research in Computer Aided Architectural Design in Europe) conference - Volumn 2 (pp. 541-546). Oulu, Finland: eCAADe and Oulu School of Architecture, University of Oulu.

Thalen, J. P. \&, van der Voort, M. C. (2012). Facilitating user involvement in product design through virtual reality. In X.-X. Tang (Ed.), Virtual reality: Human computer interaction (pp. 105-124). Rijeka, Croatia: InTech.

Whyte, J., \& Nikolić, D. (2018). Virtual reality and the built enviornment. Abingdon, Oxon : Routledge.

Author Bios:

Yi Lin Wong is a Research Assistant Professor from the School of Design at the Hong Kong Polytechnic University. Her research interests include design psychology, inclusive design, participatory design, public design, creativity and design education.

Chi Hang Lo is a Lecturer in Hong Kong Design Institute. His research interests include inclusive design, waste management and interior design for the disabled and the deprived.

Acknowledgements: The authors wish to acknowledge the support provided by the Hong Kong Polytechnic University for the data collection and preparation of the paper. 